\title{
Mobile Real-time Tracking of Acute Stroke Patients and Instant, Secure Inter-team Communication - the Join App
}

\author{
Stephan A. Munich, MD', Lee A. Tan, MD', Danilo M. Nogueira, MD', Kiffon M. Keigher, ACNP', \\ Michael Chen, MD', R. Webster Crowley, MD', James J. Conners, MD, MS², Demetrius K. Lopes, MD'
}

Purpose: The primary correlate to survival and preservation of neurologic function in patients suffering from an acute ischemic stroke is time from symptom onset to initiation of therapy and reperfusion. Communication and coordination among members of the stroke team are essential to maximizing efficiency and subsequently early reperfusion. In this work, we aim to describe our preliminary experience using the Join mobile application as a means to improve interdisciplinary team communication and efficiency.

Materials and Methods: We describe our pilot experience with the initiation of the Join mobile application between July 2015 and July 2016. With this application, a mobile beacon is transported with the patient on the ambulance. Transportation milestone timestamps and geographic coordinates are transmitted to the treating facility and instantly communicated to all treatment team members. The transport team / patient can be tracked en route to the treating facility.

Results: During our pilot study, 62 patients were triaged and managed using the Join application. Automated time-stamping of critical events, geographic tracking of patient transport and summary documents were obtained for all patients. Treatment team members had an overall favorable impression of the Join application and recommended its continued use.

Conclusion: The Join application is one of several components of a multi-institutional, interdisciplinary effort to improve the treatment of patients with acute ischemic stroke. The ability of the treatment team to track patient transport and communicate with the transporting team may improve reperfusion time and, therefore, improve neurologic outcomes.

Key Words : Mobile smartphone application; Acute ischemic stroke management;

Acute ischemic stroke workflow

'Department of Neurosurgery, Rush University Medical Center, Chicago, IL

2Department of Neurology, Rush University Medical Center, Chicago, IL

Received April 21, 2017; Revised July 7, 2017; Accepted July 12, 2017.

Correspondence to: Demetrius K. Lopes, MD, Department of Neurosurgery, Rush University Medical Center, 1725 W. Harrison, Suite 855 , Chicago, IL 60612

Tel. 312-942-1854 Fax. 312-942-2176

This is an Open Access article distributed under the terms of the Creative Commons Attribution Non-Commercial License (http://creativecommons.org/licenses/by-nc/3.0) which permits unrestricted non-commercial use, distribution, and reproduction in any medium, provided the original work is properly cited. 
Technologic advances in therapeutic strategies for the treatment of ischemic conditions, such as myocardial infarction and ischemic stroke, have markedly improved outcomes. However, advancement and development of the infrastructure housing these therapeutic approaches are critical, yet often overlooked, components of providing optimal care for patients suffering these conditions. To address this need, our institution has designed and implemented a multidisciplinary and multi-institutional workflow and infrastructure to support and streamline the treatment of patients with acute ischemic stroke. One novel component of this process is our implementation of a mobile smartphone software application that allows patient tracking and secure communication among treatment team members.

More than two-thirds of physicians regularly use smartphones [1]. Mobile smartphone applications are being used broadly in neurological disease management, spanning from disease prevention to tracking of longterm follow-up [2]. The use of mobile applications has been utilized in a variety of ways for the management of acute ischemic stroke - digital health interventions for the reduction of vascular risk factors and prevention of cardiovascular diseases [3, 4], diagnosis of stroke at telestroke centers [5], calculation of NIH stroke scores and ASPECTS scores [6], and automated assessment of modified Rankin scale after discharge [7].

The utilization of mobile smartphone technology in the triage and management of patients with acute ischemic stroke may further streamline management of these patients. Here, we describe our experience implementing the Join smartphone application at our comprehensive stroke center. We aim to describe the impact of the application on our workflow and report the impressions of our team in implementing this application.

\section{MATERIALS AND METHODS}

\section{Clinical Workflow for Acute Ischemic Stroke Triage and Management}

Our "Stroke-60" acute ischemic stroke workflow typically begins with a call from an outside facility where there is a patient in whom ischemic stroke is suspected (Fig. 1). The stroke neurologist at our institution discusses the patient and reviews available imaging. He/she discusses the case with the neurointerventionalist and the decision to transfer the patient for endovascular intervention is made. If the patient meets criteria for intravenous thrombolytics, it is typically administred at the outside facility and continued during transport (i.e. "drip and ship" model). Upon an affirmative decision to initiate transfer, the stroke team (i.e. stroke neurology and neurosurgery services, anesthesia team, nurse, and radiation technologist) is notified via page. The neuroscience intensive care unit charge nurse is also notified to facilitate timely post-

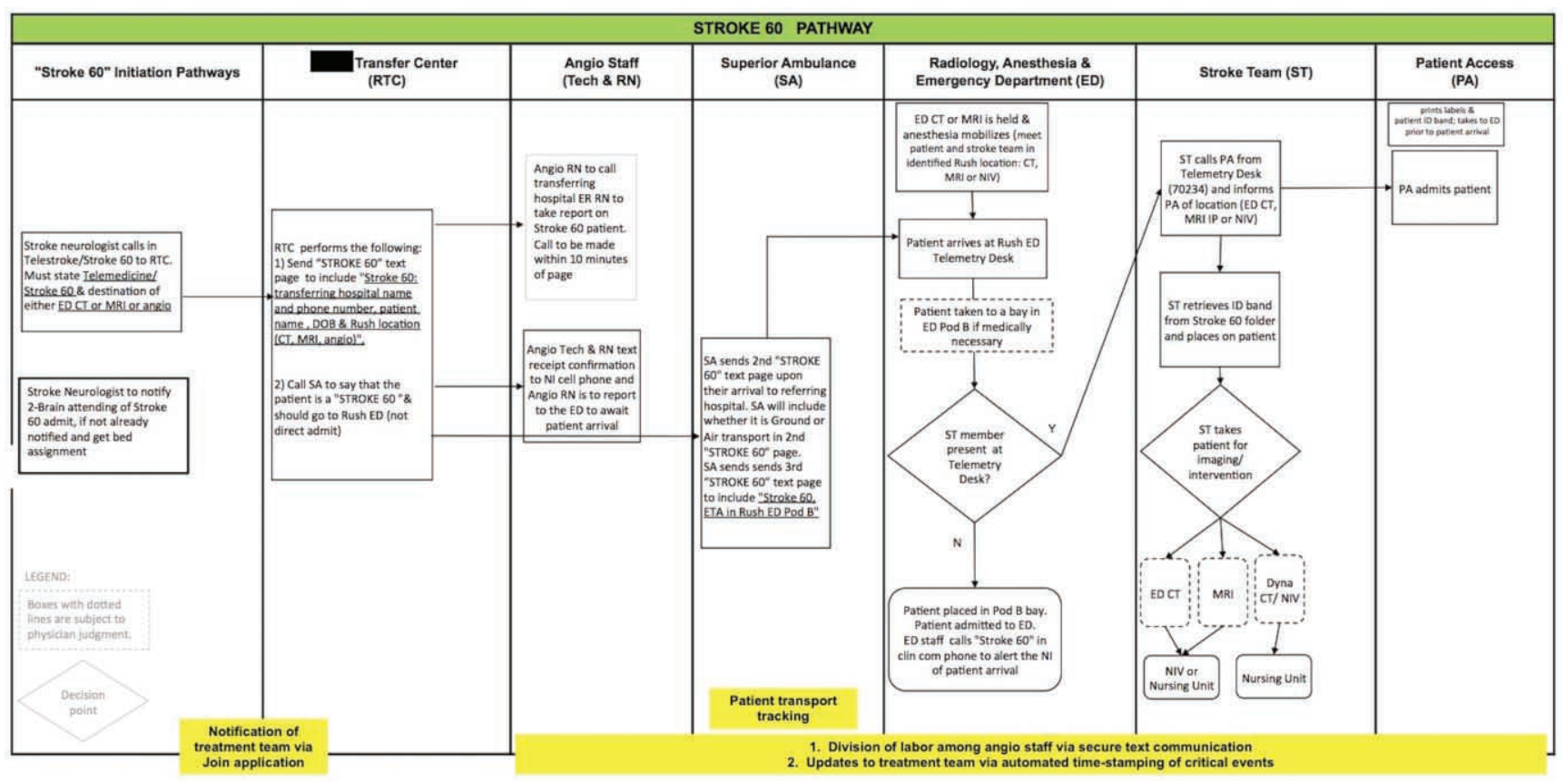

Fig. 1. Stroke 60 Workflow Algorithm. The Join application has been integrated into our Stroke 60 workflow. 
intervention admission. A series of subsequent pages are sent to the stroke team as additional information becomes available - confirmation of patient name and date of birth, departure time from the outside facility, estimated time of arrival at our facility.

The patient arrives at the emergency room, bypassing the ED triage bay, where he/she is met by the neuroendovascular and stroke neurology teams and is taken directly to the imaging suite. A rapid neurologic assessment, including determination of NIHSS, is performed en route to the $\mathrm{CT}$ scanner where non-contrast head $\mathrm{CT}$, CTA, with/without CT perfusion are obtained. Whether to proceed to the neuro-endovascular suite is determined based upon clinical and radiographic assessment. If the patient is deemed a candidate for intervention, he/she is escorted directly to the neuroendovascular suite where the anesthesia team and nursing staff are waiting. Thrombectomy procedures were routinely performed under conscious sedation, unless there was concern for airway protection. In cases with a compromised airway or a mental status that precluded safe administration of conscious sedation, the anesthesia team performed general anesthesia.

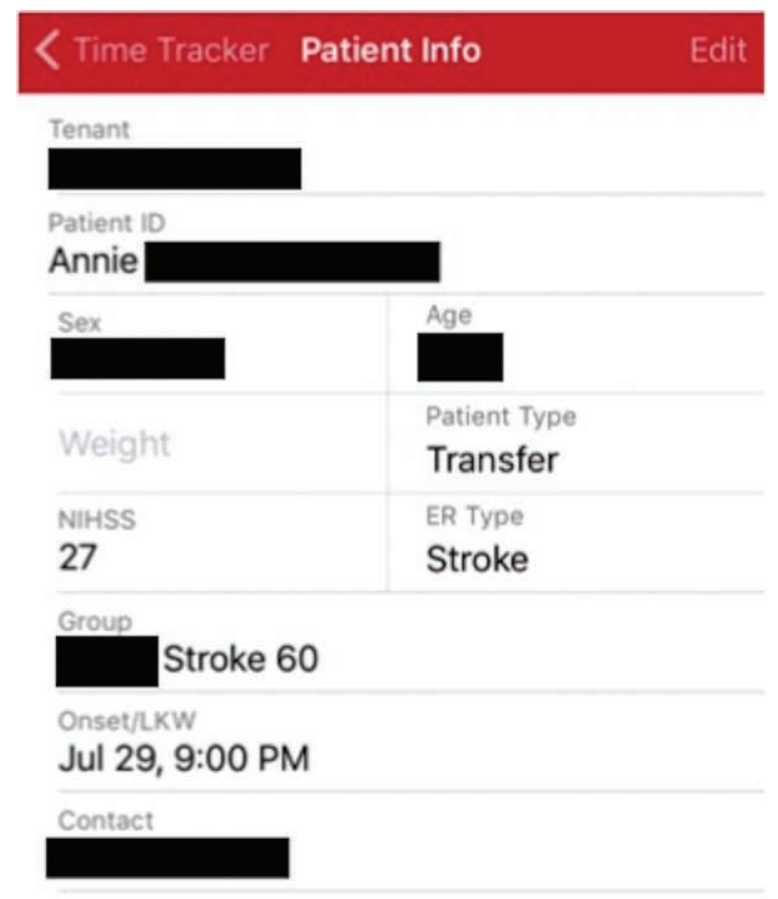

Start Tracking View Location

Fig. 2. Initial notification that is sent to the entire treatment team. This notification contains basic demographic and contact information, as well as time of onset (or last known well).

\section{Patient Population}

During our pilot study (July 2015 through July 2016), 62 patients were triaged and managed using the Join application. Use of the Join application during this time period was at the discretion of the physician. Patients captured in this study include patients that were transferred from other hospitals, as well as those presenting to our emergency room. Since the pilot study, we routinely triage and manage all acute stroke patients using the Join application. IRB approval was not required for this study.

\section{Join Mobile Smartphone Application}

A customized mobile smartphone application, "Join" (Allm, Inc, Tokyo, Japan) was developed in an effort to streamline our Stroke-60 workflow model described above. Important features of this application include:

1. Immediate notification to the entire treatment team of basic patient information and demographics (Fig. 2)

2. Automated time-stamping of events

3. Real-time, secure communication among treatment team members

4. HIPPA compliant transmission of patient demographics and clinical information

5. Real-time monitoring and communication of the patient's exact position during transport using global positioning system (GPS)

6. Generation of a summary document in portable document format (PDF) format that can be uploaded into the electronic medical record

Time-stamped events include: time of onset (or last known well), time of IV-tPA administration, time consent was obtained, time of arrival (door time), time of completion of imaging, arrival to the angiography suite, procedure start time (groin puncture), first pass time, and recanalization time. These events and their timestamps are recorded by a member of the treatment team (usually a nurse practitioner) and are immediately visible to the treatment team via the smartphone application (Fig. 3).

Given the multiple stages of patient transport and the unavoidable obstacles due to our location in a major metropolitan area (e.g. rush hour traffic, detours, etc.), incorporation of a real-time GPS-based patient tracking system was developed to inform treatment team members of the transport progress (Fig. 4). The moment that the transporting team logs into their mobile version of the Join application, all the stroke team members can follow the ambulance en route to the hospital - reminiscent of an "Uber" ride request. 
After completion of the endovascular intervention and admission to the ICU, the information is transmitted in the form of a summary document. It can be available by PDF for printing and/or upload into the

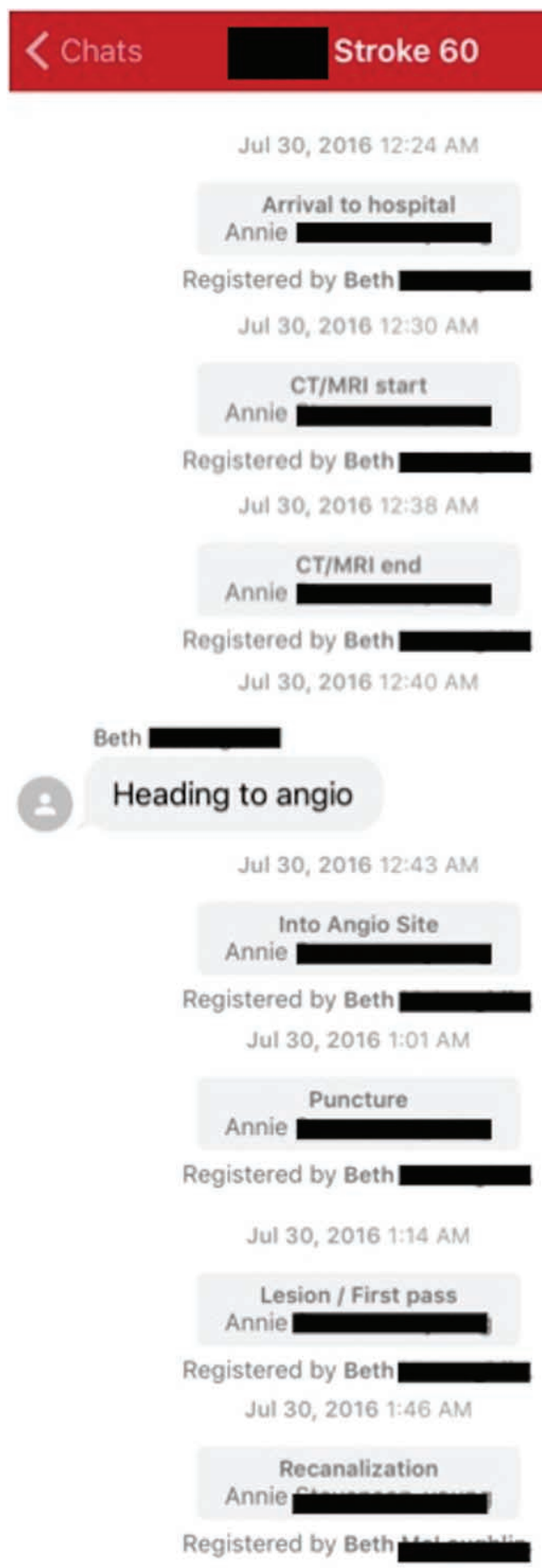

Fig. 3. Chat thread demonstrating time-stamped critical events during patient management and triage. These time stamps can be viewed by the entire treatment team. Members of the treatment team can also notify other members of the patient progress, in this case that the imaging was favorable for endovascular intervention. electronic medical record as a stroke code report.

\section{Assessment of Join Application}

Following our pilot trial of 62 patients, we administered a survey (questions listed in Table 1) to the treatment team members to determine the usability of the application. The survey was administered to the EMS personnel, radiology technologists, nurses, research personnel, advanced practice providers and physicians.

\section{RESULTS}

During this pilot trial, we triaged and managed 62 patients using the Join application. We found that the secure text communication feature facilitated the rapid delegation of tasks and ensured confirmation of their completion among the entire team. Additionally, this feature allowed for communication of deviations from the protocols (so called, "audibles") when they were necessary due to various circumstances.

Automated time-stamping of events was performed for all patients. Summary reports also were obtained for all patients and successfully uploaded to the electronic medical record.

Following the pilot trial of the Join application we administered surveys to the treatment team members to assess the applications usability. The survey found a generally positive impression of the Join application (Table 1 and Fig. 5). Of note, $87.5 \%$ of respondents found the application easy to use and $82.5 \%$ of respondents recommended continuing to use the application as a method of team communication.

\section{DISCUSSION}

To our knowledge, this is the first report describing the integration of a mobile, smartphone application with GPS (patient tracking) functionality in the workflow and management of acute ischemic stroke patients. The Join application described here coordinates the activities and travel logistics of all facets of this workflow, including the patient, initial presenting hospital, transportation service (ambulance or helicopter), receiving hospital, associated physicians and on-call treatment team members. This secure application is HIPPA compliant and able to capture data that subsequently can be integrated as a permanent part of the patient's electronic medical record.

Smartphones increasingly are becoming a part of our personal and professional lives, with more than two- 
thirds of physicians reporting their regular use. The use of smartphones and their mobile applications have similarly been increasing in the realm of acute ischemic stroke. To date, mobile applications have primarily been used in the setting of risk factor monitoring and management [3], assessment of stroke severity (via
NIHSS) [8], and rehabilitation following stroke [9, 10]. While GPS technology has been used in stroke-focused mobile applications previously, it has only been used in a static way, locating the nearest treatment center [11]. Here, we present the first report of a mobile smartphone application designed to track patient location

Table 1. Survey Administered to Users of the Join Application

\begin{tabular}{ll}
\hline 1 & I like the concept of the Join application \\
2 & I have obtained information about transfers via Join earlier than the traditional method of pages/calls \\
3 & I find that I have less downtime waiting for the patient due to the ability to track patient location \\
4 & Information has been reliable \\
\hline 5 & I feel the data collected in Join should be made a permanent part of the patient medical record \\
6 & It is an easy-to-use application \\
7 & It adds extra work to an existing process \\
\hline 8 & Information technology support is always accessible for problems that arise \\
9 & I recommend continuing this method of team communication \\
\hline
\end{tabular}

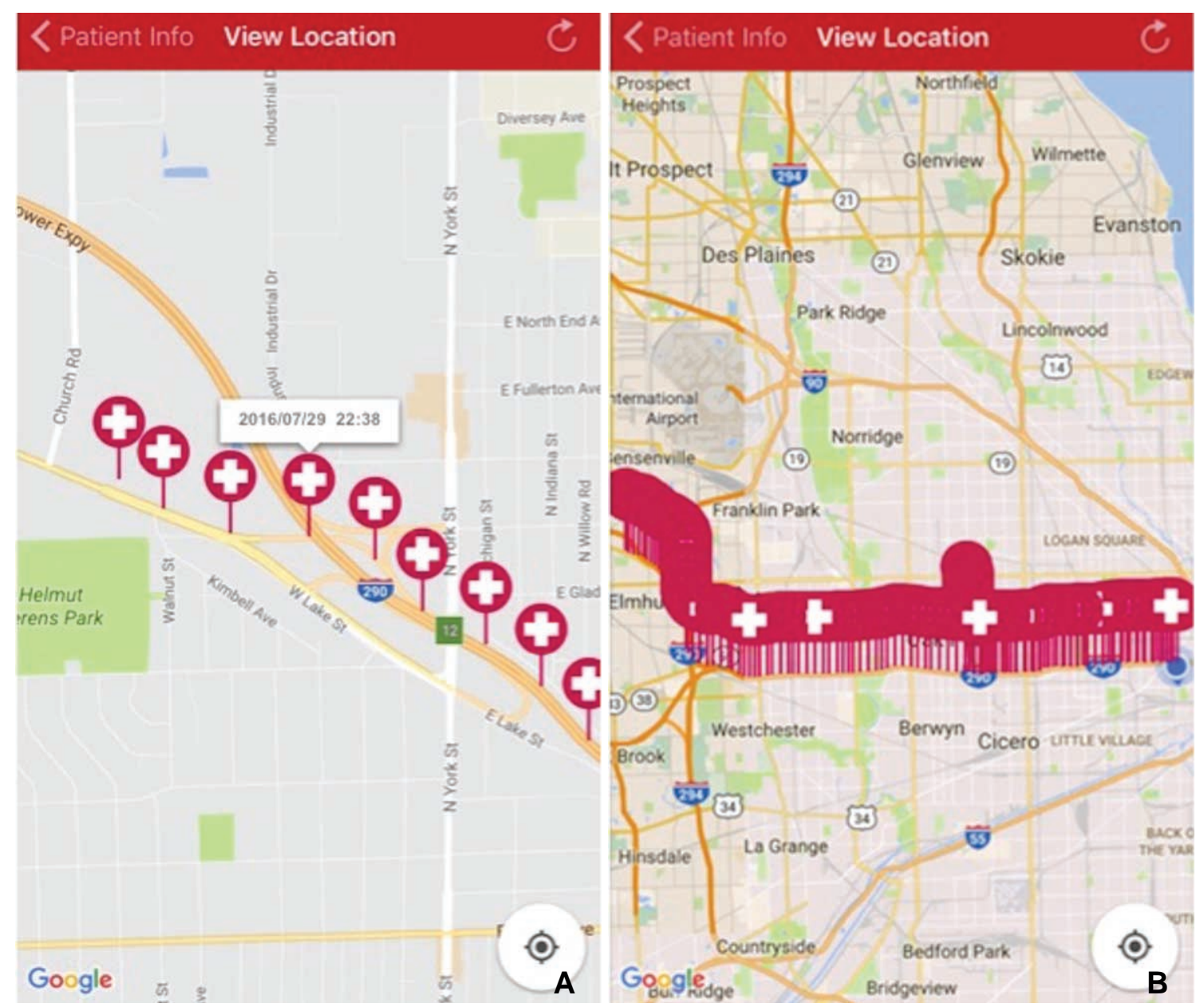

Fig. 4. Real-time patient tracking feature of the Join application. The patient's pick-up time and location can be seen (A). Transport to the hospital can also be tracked in real-time (B). 


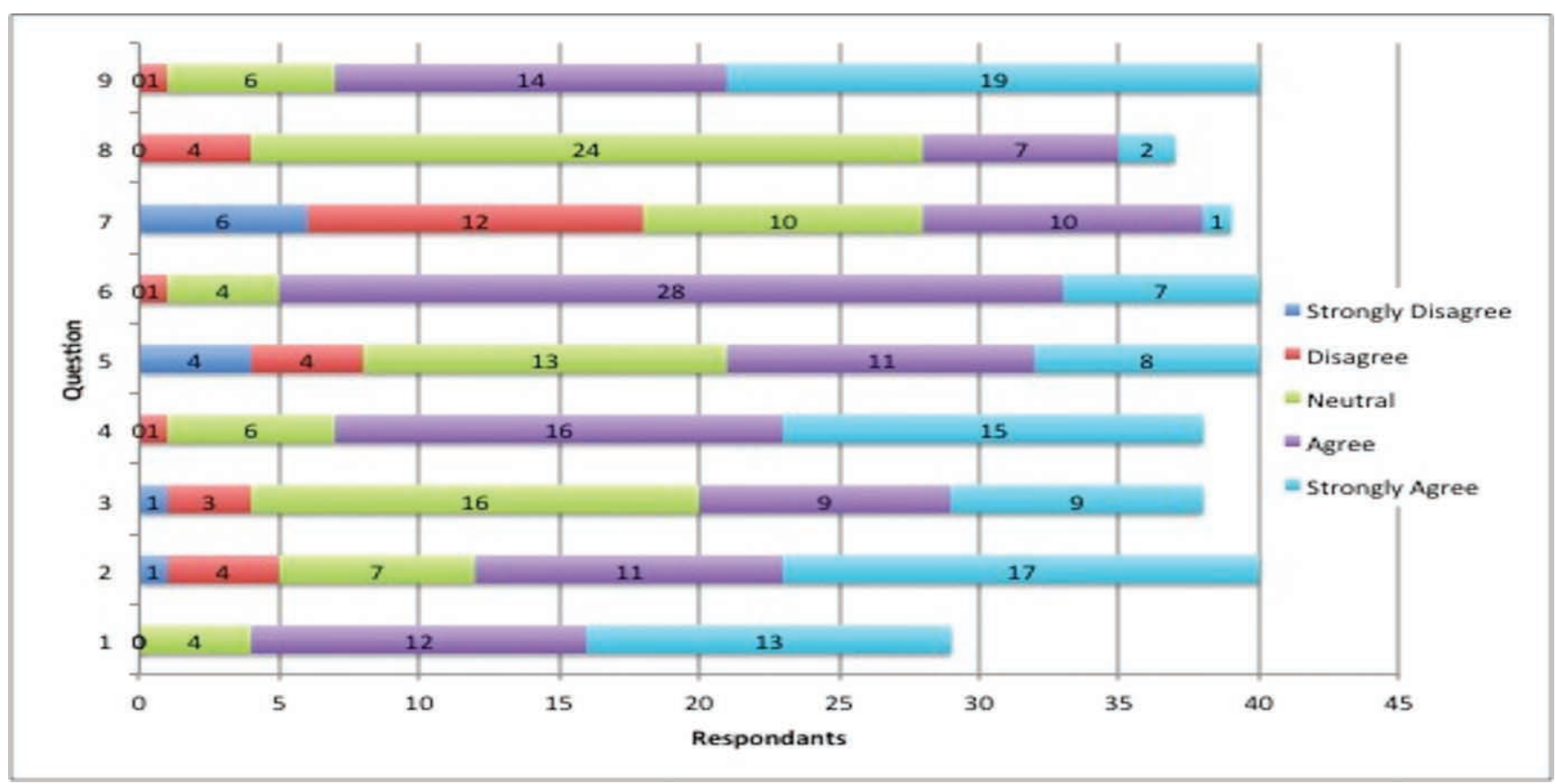

Fig. 5. Results of survey administered to physicians, nurse practitioners, nurses, radiation technologists, and EMS personnel using the Join application. Questions are listed below:

1. I like the concept of the Join application

2. I have obtained information about transfers via Join earlier than the traditional method of pages/calls.

3. I find that I have less downtime waiting for the patient due to the ability to track patient location.

4. Information has been reliable.

5. I feel the data collected in Join should be made a permanent part of the patient medical record.

6. It is an easy-to-use application.

7. It adds extra work to an existing process.

8. Information technology support is always accessible for problems that arise.

9. I recommend continuing this method of team communication.

thereby helping to coordinate immediate endovascular treatment and management of acute ischemic stroke.

One potential limitation of this application is that it requires a team member to record data points in real time in the application. Although this is an added responsibility to one of the team members, our team did not perceive that this responsibility created any obstacle to patient care. Rather the Join application interface allows for recording of these time points with a simple click on the smart phone screen, an action that typically takes a fraction of a second.

As seen from the results of the administered survey, the response to implementation of the Join application was generally positive. Of the groups surveyed, EMS personnel rated the application the less favorably compared to hospital-based team members. Of EMS personnel surveyed, $52.4 \%$ reported that the application added extra work to the existing process. We hypothesize that this response may have been the foundation for an overall less positive response from this group. Despite this finding, $95.2 \%$ of EMS personnel felt the application was easy to use and $85.7 \%$ recommended continued use of the application, which was actually slightly higher than the group as a whole. One potential reason that they largely favored continued use of the application may be the ability to relay results from the hospital course, such as imaging and treatment, through the application. For example, we routinely transmit a picture of the retrieved thrombus through the application, which allows all members to see the results of their efforts. This is of particular benefit to the EMS personnel, who can often be left in the dark regarding a patient's outcome following transport. Instead, through communication with Join, the findings and results of the patient's treatment can be relayed to the entire team.

An additional benefit of the Join application is its inherent reduction in hand-offs. Since communication is transmitted to a text message thread that is available to the entire treatment team, there is decreased need for hand-offs between individual members of the team. Hand-offs are often necessary in patient care settings, but it is well-recognized that higher number of hand- 
offs are associated with an increased incidence of medical errors. 12 The ability to reduce, or eliminate, the number of hand-offs will likely improve the efficiency and safety of care.

As those treating acute ischemic stroke know well, an effective and efficient treatment team is critical to the delivery of care. One, often overlooked aspect, of this is physician and team burnout. While the concept of physician burnout has become more recognized, burnout associated specifically with those treating acute ischemic stroke is less well-studied. In a Japanese survey of 2,564 physicians working for acute stroke care, the number of hours worked per week and the time spent in stroke care were positively associated with the severity of burnout.13 Interestingly, the study also found that burnout was less likely in accredited hyperacute stroke centers. The authors attribute this finding to the fact that these institutions had sufficient staffing, as required by the Japanese Joint Commissions.

We believe the Join application will help alleviate treatment team burnout largely by maximizing time efficiency. Through instantaneous notification of patient location, the treatment team can time necessary preparations for the patient's arrival, including their own arrival at the hospital, rather than waiting idle for an indefinite amount of time. Task assignment through secure messaging allows team members to "divide and conquer," improving efficiency of workflow, and reducing duplication of tasks such as calling the family for consent or screening for clinical trial inclusion. Secure messaging with EMS personnel permits communication with the transporting team and allows the team to be prepared for any changes in patient condition that may occur during transport. Additionally, for those patients who do not end up needing endovascular therapy, communication through Join instantly alerts all members of the team that the procedure has been cancelled, minimizing the amount of time spent in the hospital.

Lastly, while the application helps with efficiency, the ability to remotely follow a case to its conclusion may also aid with reducing burnout. In essence, it permits the entire team to see the "fruits of their labor" regardless of whether or not they remain involved with the patient's care. In a busy comprehensive stroke center such as ours, team members may be called in after hours as many as 2 to 3 times per week. Therefore, attention to team morale and burnout is critical for the retention of staff and continued improvement in the delivery of care.
The Join application described here represents the first generation of a real-time, mobile patient tracking smartphone application. As such, there is certainly room for improvement. Our survey revealed an overall less positive response to the application by EMS personnel. Automation of EMS involvement in the application, rather than the current requirement of active sign on, may be one improvement that may improve EMS personnel perception and streamline use of the application. Additional improvements may include addition of the EMS neurologic evaluation and transport care updates into Join application time stamps.

\section{CONCLUSION}

In our initial experience, the Join application provided reliable, graphical, real-time tracking of patient location during transport, secure communication between treatment team members, and accurate time-stamping of critical events during the triage and management of patients with acute, ischemic stroke. Our pilot experience has been positive, though qualitative in nature. Additional, prospective and quantitative validation and continued work to improve the efficiency of stroke care is warranted.

\section{References}

1. Thomairy NAM MM, Alsalamah SM, Moussa N, Coustasse A. Use of smartphones in hospitals. SO - Health Care Manager. Vol 342015:297-307

2. Cohen AB NB, Sheth KN. Mobile medical applicaitons in neurology. Neurology in Clinical Practice 2013;3:52-60

3. Seo WK, Kang J, Jeon M, Lee K, Lee S, Kim JH, et al. Feasibility of using a mobile application for the monitoring and management of stroke-associated risk factors. J Clin Neurol 2015;11:142-148

4. Widmer RJ, Collins NM, Collins CS, West CP, Lerman LO, Lerman A. Digital health interventions for the prevention of cardiovascular disease: a systematic review and meta-analysis. Mayo Clin Proc 2015;90:469-480

5. Demaerschalk BM, Vargas JE, Channer DD, Noble BN, Kiernan TE, Gleason EA, et al. Smartphone teleradiology application is successfully incorporated into a telestroke network environment. Stroke 2012;43:3098-3101

6. Gonzalez MA, Hanna N, Rodrigo ME, Satler LF, Waksman R. Reliability of prehospital real-time cellular video phone in assessing the simplified National Institutes Of Health Stroke Scale in patients with acute stroke: a novel telemedicine technology. Stroke 2011;42:1522-1527

7. Cooray C, Matusevicius M, Wahlgren N, Ahmed N. Mobile Phone-Based Questionnaire for Assessing 3 Months Modified Rankin Score After Acute Stroke: A Pilot Study. Circ Cardiovasc Qual Outcomes 2015;8(6 Suppl 3):S125-130 


\section{Stephan A. Munich, et al.}

8. Demaerschalk BM, Vegunta S, Vargas BB, Wu Q, Channer DD, Hentz JG. Reliability of real-time video smartphone for assessing National Institutes of Health Stroke Scale scores in acute stroke patients. Stroke 2012;43:3271-3277

9. Choi YH, Ku J, Lim H, Kim YH, Paik NJ. Mobile game-based virtual reality rehabilitation program for upper limb dysfunction after ischemic stroke. Restor Neurol Neurosci 2016;34:455-463

10. Zhang MW, Chew PY, Yeo LL, Ho RC. The untapped potential of smartphone sensors for stroke rehabilitation and after-care. Technol Health Care 2016;24:139-143

11. Nam HS, Heo J, Kim J, Kim YD, Song JJ, Park E, et al. Development of smartphone application that aids stroke screening and identifying nearby acute stroke care hospitals. Yonsei Med J 2014;55:25-29

12. Zendejas B, Ali SM, Huebner M, Farley DR. Handing over patient care: is it just the old broken telephone game? J Surg Educ 2011;68:465-471

13. Nishimura K, Nakamura F, Takegami M, Fukuhara S, Nakagawara J, Ogasawara K, et al. Cross-sectional survey of workload and burnout among Japanese physicians working in stroke care: the nationwide survey of acute stroke care capacity for proper designation of comprehensive stroke center in Japan (JASPECT) study. Circ Cardiovasc Qual Outcomes 2014;7:414422 